\title{
A case of lymphomatoid granulomatosis masquerading as a lung abscess
}

\author{
M McCloskey, M Catherwood, D McManus, G Todd, R Cuthbert, M Riley
}

Thorax 2004;59:818-819. doi: 10.1136/thx.2003.008094

Lymphomatoid granulomatosis (LG) is a rare T cell rich, B cell non-Hodgkin's lymphoma which is difficult to diagnose. We present a patient with LG who demonstrated many of the difficulties in diagnosis and highlighted the importance of reviewing the diagnosis if treatment does not have the anticipated effect.

A 49 year old male smoker presented to another hospital with a 3 month history of weight loss and sweats. A chest radiograph showed a mass in the left lower lobe and bronchoscopic examination revealed inflammation involving the left lower lobe bronchus. Washings were negative for organisms and malignancy. A computed tomographic (CT) scan showed a cavitating mass in the left lower lobe with multiple smaller opacities throughout both lung fields. Percutaneous fine needle aspiration of the mass showed inflammation with necrotic debris. He was discharged but presented 1 week later with fever and rigors and was transferred to this hospital.

On arrival he had a cough productive of copious, foul smelling, purulent sputum. His temperature was $40^{\circ} \mathrm{C}$. There was no lymphadenopathy or hepatosplenomegaly. Neurological examination was normal. C-reactive protein (CRP) was $59 \mathrm{mg} / \mathrm{l}$. Blood, urine and sputum cultures, white cell count, serum angiotensin converting enzyme, $\mathrm{P}$ - and $\mathrm{C}$ ANCA, autoantibody screen, complement, test for HIV, viral screening, and brucella titres were all negative or normal. A repeat bronchoscopic examination was non-diagnostic. A repeat CT scan showed a $7 \mathrm{~cm}$ cavitating lesion in the left lower lobe with an air/fluid level and minor "inflammatory changes" in the right lung.

Empirical treatment for a suspected lung abscess was commenced using intravenous benzylpenicillin, gentamicin, and metronidazole. Over the next 21 days his pyrexia settled, sputum cleared, and the CRP level normalised. The chest radiograph remained unchanged. Four weeks later his purulent sputum, fever, and raised CRP level recurred. Open lung biopsy was considered, but the thoracic surgical team declined because of the evidence for active infection. Antibacterial therapy was recommenced with intravenous cefotaxime and metronidazole. As before, there was a good clinical response but without radiological resolution. Further symptomatic relapse occurred once the drugs were withdrawn. Seven months after initial presentation he deteriorated further. A CT scan showed the left lower lobe cavity unchanged but there were now numerous nodular densities throughout the right lung (fig 1). Repeat bronchial biopsies showed abnormal lymphoid tissue characterised by a polymorphous lymphoid infiltrate containing scattered enlarged blast-like cells. These stained positively for CD20, a B cell marker, and immunoglobulin rearrangement studies showed clonality. These findings suggested lymphomatoid granulomatosis.
He was treated with methylprednisolone, vinblastine, and cyclophosphamide. Within 2 weeks his dyspnoea, fever, and right sided radiological abnormalities resolved. The left lower lobe lesion reduced by $40 \%$ after three cycles of chemotherapy. However, he continued to complain of purulent foul smelling sputum. After a fourth cycle of chemotherapy he developed presumed invasive candidiasis and died. The family declined a post mortem examination.

\section{DISCUSSION}

Liebow et al first described lymphomatoid granulomatosis (LG) as a triad of polymorphic lymphoid infiltrate, angiitis, and granulomatosis. ${ }^{1}$ It is thought to represent a clonal proliferation of $\mathrm{B}$ cells showing angiocentric growth and destructive behaviour. It is similar to post-transplant lymphoproliferative disorders in that Epstein-Barr virus (EBV) is frequently found. Histological diagnosis may be problematic. ${ }^{2}$ Differentiation from high grade non-Hodgkin's lymphoma is aided by the presence of angiocentricity and EBV infection. Wegener's granulomatosis may have a similar appearance. ${ }^{3}$ In our patient in situ hybridisation for EBV was difficult to interpret because of the small samples.

As in our case, the typical patient is a male smoker in his fifth decade. Presenting features include cough, haemoptysis, and chest pain. Skin rashes and peripheral neuropathy may occur. Lesions may "wax and wane" and delay in diagnosis is common. ${ }^{4}$ Bronchoscopic biopsy is positive in $27 \%$ of cases, but open lung biopsy is usually positive and is the diagnostic method of choice. No well studied treatment exists, but prednisolone, cyclophosphamide, and more aggressive chemotherapy are generally used. The prognosis is poor. ${ }^{4}$ As a result of the similarities between EBV associated post-transplant lymphoma and LG, interferon $\alpha 2 \mathrm{~b}$ has been tried because of its antiviral, antiproliferative,

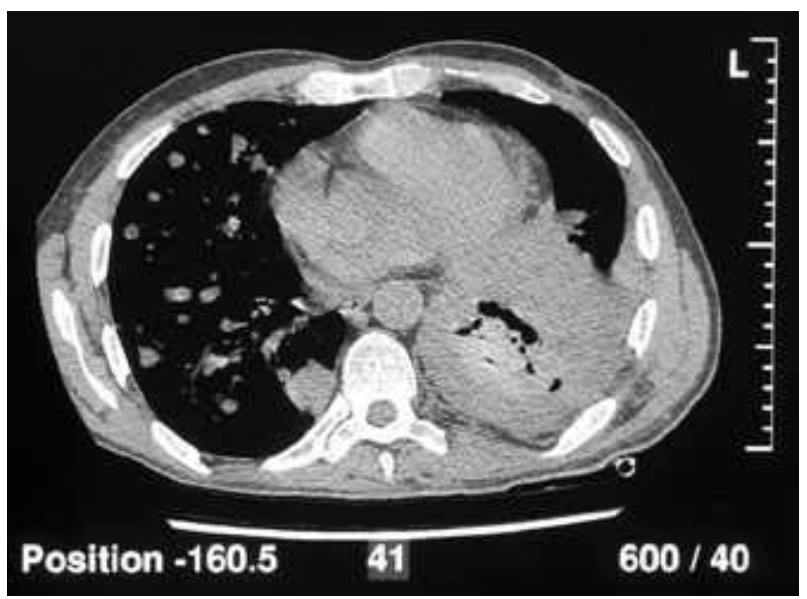

Figure 1 CT scan showing a cavitating lesion in the left lower lobe and multiple opacities in the right lung. 
and immunomodulatory effects. ${ }^{5}$ Bone marrow transplantation was successful in one case. ${ }^{6}$

The partial response to antibacterial therapy in our case remains puzzling. We are not aware of previous reports of the benefit of antibacterial therapy in LG. No significant bronchial obstruction was identified to suggest post-obstructive pneumonia and there was nothing in the history to suggest aspiration. The symptoms suggestive of lung abscess may not have been caused by infection. Despite repeated sampling, no organism was ever cultured. In Wegener's granulomatosis chronic antigen load from infection may be aetiologically important, and there is some evidence that cotrimoxazole may improve the nasal manifestations. ${ }^{7}$ A direct immunomodulatory effect of antibacterial therapy is another potential mechanism that could explain the clinical response in our case. ${ }^{8}$ LG has been associated with diseases manifesting impaired $\mathrm{T}$ cell function such as acquired immune deficiency syndrome ${ }^{9}$ and Sjogren's syndrome. ${ }^{10}$ HIV testing was negative in our patient. We did not undertake formal tests of $\mathrm{T}$ cell function and a $\mathrm{T}$ cell defect remains a possible cause for the septic picture.

In our patient the presentation with features suggesting lung abscess and the initial response to treatment strongly dissuaded the thoracic surgical team from intervening. Diagnosis was almost certainly delayed as a result. This case demonstrates the difficulties that may be faced in diagnosing LG, particularly without open lung biopsy, and underscores the importance of reviewing the diagnosis if treatment does not have the anticipated effect.

\section{ACKNOWLEDGEMENTS}

The authors thank Dr A U Wells of the Royal Brompton Hospital for his most helpful discussion of the case.

\section{Authors' affiliations \\ M McCloskey, M Catherwood, D McManus, G Todd, R Cuthbert, \\ M Riley, Belfast City Hospital, Belfast, Northern Ireland, UK}

Correspondence to: $\operatorname{Dr} M M M c C l o s k e y$, Altnagelvin Area Hospital, Glenshane Road, Londonderry, Northern Ireland, UK;

mccloskeymargaret@hotmail.com

Received 9 April 2003

Accepted 9 October 2003

\section{REFERENCES}

1 Liebow AA, Carrington CR, Friedman PJ. Lymphomatoid granulomatosis. Hum Pathol 1972;3:457-8.

2 Pisani RJ, DeRemee RA. Clinical implications of the histopathologic diagnosis of pulmonary lymphomatoid granulomatosis. Mayo Clinic Proc 1990:65:151-63.

3 Nicholson AG. Pulmonary lymphoproliferative disorders (review). Curr Diagn Pathol 2000;6:130-9.

4 Cadranel J, Wislez M, Antoine M. Primary pulmonary lymphoma. Eur Respir J 2002;20:750-62.

5 Wilson WH, Kingma DW, Raffeld M, et al. Association of lymphomatoid granulomatosis with Epstein Barr viral infection of $B$ lymphocytes and response to interferon-alpha 2b. Blood 1996;87:4531-7.

6 Bernstein ML, Reece $E$, de Chadarevian JP, et al. Bone marrow transplantation in lymphomatoid granulomatosis. Report of a case. Cancer 1986;58:969-72.

7 De Remee RA. The treatment of Wegener's granulomatosis with trimethoprimsulphamethaoxazole; illusion or vision. Arthritis Rheum 1988;31:1068.

8 Krehmeier U, Bardenhever M, Voggenreiter G, et al. Effects of antimicrobial agents on spontaneous and endotoxin-induced cytokine release of human peripheral blood mononuclear cells. J Infect Chemother 2002;8:194-7.

9 Anders $\mathrm{KH}$, Latta H, Chang BS, et al. Lymphomatoid granulomatosis and malignant lymphoma of the central nervous system in the acquired immunodeficiency syndrome. Hum Pathol 1989;20:326-34.

10 Weisbrot IM. Lymphomatoid granulomatosis of the lung associated with a long history of benign lymphoepithelial lesions of the salivary glands and lymphoid interstitial pneumonitis. Report of a case. Am J Clin Pathol 1976;66:792-801. 